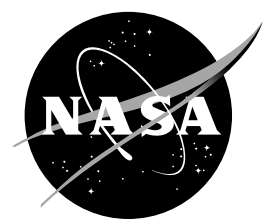

\title{
Analysis of Three-Dimensional, Nonlinear Development of Wave-Like Structure in a Compressible Round Jet
}

Milo D. Dahl

Glenn Research Center, Cleveland, Ohio

Reda R. Mankbadi

Embry-Riddle Aeronautical University, Daytona Beach, Florida 
Since its founding, NASA has been dedicated to the advancement of aeronautics and space science. The NASA Scientific and Technical Information (STI) Program Office plays a key part in helping NASA maintain this important role.

The NASA STI Program Office is operated by Langley Research Center, the Lead Center for NASA's scientific and technical information. The NASA STI Program Office provides access to the NASA STI Database, the largest collection of aeronautical and space science STI in the world. The Program Office is also NASA's institutional mechanism for disseminating the results of its research and development activities. These results are published by NASA in the NASA STI Report Series, which includes the following report types:

- $\quad$ TECHNICAL PUBLICATION. Reports of completed research or a major significant phase of research that present the results of NASA programs and include extensive data or theoretical analysis. Includes compilations of significant scientific and technical data and information deemed to be of continuing reference value. NASA's counterpart of peerreviewed formal professional papers but has less stringent limitations on manuscript length and extent of graphic presentations.

- TECHNICAL MEMORANDUM. Scientific and technical findings that are preliminary or of specialized interest, e.g., quick release reports, working papers, and bibliographies that contain minimal annotation. Does not contain extensive analysis.

- CONTRACTOR REPORT. Scientific and technical findings by NASA-sponsored contractors and grantees.
- CONFERENCE PUBLICATION. Collected papers from scientific and technical conferences, symposia, seminars, or other meetings sponsored or cosponsored by NASA.

- SPECIAL PUBLICATION. Scientific, technical, or historical information from NASA programs, projects, and missions, often concerned with subjects having substantial public interest.

- TECHNICAL TRANSLATION. Englishlanguage translations of foreign scientific and technical material pertinent to NASA's mission.

Specialized services that complement the STI Program Office's diverse offerings include creating custom thesauri, building customized data bases, organizing and publishing research results ... even providing videos.

For more information about the NASA STI Program Office, see the following:

- Access the NASA STI Program Home Page at http://www.sti.nasa.gov

- E-mail your question via the Internet to help@sti.nasa.gov

- Fax your question to the NASA Access Help Desk at 301-621-0134

- Telephone the NASA Access Help Desk at 301-621-0390

- Write to:

NASA Access Help Desk

NASA Center for AeroSpace Information 7121 Standard Drive

Hanover, MD 21076 
NASA/TM-2002-211585

AIAA-2002-2451

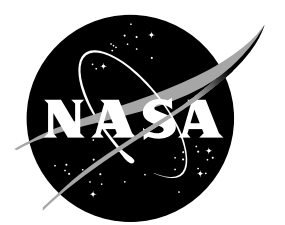

\section{Analysis of Three-Dimensional, Nonlinear Development of Wave-Like Structure in a Compressible Round Jet}

Milo D. Dahl

Glenn Research Center, Cleveland, Ohio

Reda R. Mankbadi

Embry-Riddle Aeronautical University, Daytona Beach, Florida

Prepared for the

Eighth Aeroacoustics Conference

cosponsored by the American Institute of Aeronautics and Astronautics and the Confederation of European Aerospace Societies

Breckenridge, Colorado, June 17-19, 2002

National Aeronautics and

Space Administration

Glenn Research Center 
The Aerospace Propulsion and Power Program at NASA Glenn Research Center sponsored this work.

Available from

NASA Center for Aerospace Information 7121 Standard Drive

Hanover, MD 21076
National Technical Information Service 5285 Port Royal Road Springfield, VA 22100

Available electronically at http://gltrs.grc.nasa.gov/GLTRS 


\title{
ANALYSIS OF THREE-DIMENSIONAL, NONLINEAR DEVELOPMENT OF WAVE-LIKE STRUCTURE IN A COMPRESSIBLE ROUND JET
}

\author{
Milo D. Dahl* \\ National Aeronautics and Space Administration \\ Glenn Research Center \\ Cleveland, Ohio 44135 \\ Reda R. Mankbadi ${ }^{\dagger}$ \\ Embry-Riddle Aeronautical University \\ Daytona Beach, Florida 32114
}

\begin{abstract}
An analysis of the nonlinear development of the large-scale structures or instability waves in compressible round jets was conducted using the integral energy method. The equations of motion were decomposed into two sets of equations; one set governing the mean flow motion and the other set governing the large-scale structure motion. The equations in each set were then combined to derive kinetic energy equations that were integrated in the radial direction across the jet after the boundary-layer approximations were applied. Following the application of further assumptions regarding the radial shape of the mean flow and the large structures, equations were derived that govern the nonlinear, streamwise development of the large structures. Using numerically generated mean flows, calculations show the energy exchanges and the effects of the initial amplitude on the coherent structure development in the jet.
\end{abstract}

\section{INTRODUCTION}

Strong evidence suggests that jet noise, particularly in the supersonic regime, is dominated by contributions from wave-like structures in the initial region in the jet that are associated with Mach-wave radiation. These structures cannot be captured by classical turbulence modeling. Direct Numerical Simulations (DNS) and Large Eddy Simulations (LES) can successfully capture these structures, but they are computationally intensive.

There have been several attempts to model this structure using the integral energy approach. The basic idea is that the coherent structure is modeled as nonlinear instability waves that grow and decay along the jet. Each flow component is split into three quantities: a time-average mean component, a large-scale wave-like coherent component, and a fine-scale random turbulence component. Starting from the full compressible Navier-Stokes equations and using time averaging, the momentum equations for each flow

\footnotetext{
${ }^{*}$ Research Scientist, Member AIAA

${ }^{\dagger}$ Professor \& Department Chair, Associate Fellow AIAA
}

component can be obtained. These equations are then used to derive the kinetic energy equations, which are integrated across the jet to produce a set of simultaneous, ordinary differential equations describing the interactions among the various scales of motion. Shape assumptions are then made to close these equations to obtain the development of each flow component. The plane shear layer case has been studied by several researchers. ${ }^{1,2}$ The incompressible round jet case has been studied for single and multi-frequency modes. ${ }^{3,4}$ The compressible planar shear layer case has been studied by Lee \& Liu. ${ }^{5}$ The focus of this study is the compressible, supersonic round jet case because of its obvious relevance to practical applications.

Apart from modeling the physics involved, the study of the development of the coherent mode was conducted to simulate excited jets. Excitation via single or multifrequency mode has been conducted in the past to control the jet signature. For instance, Raman et al. ${ }^{6}$ show that by exciting an incompressible round jet via forced waves of various frequencies, amplitudes, and phases, the jet can be made to spread faster and thus may reduce its noise signature. Arbey \& Ffowcs-Williams ${ }^{7}$ considered the objective of reducing the peak noise by forcing an excitation wave at the subharmonic frequency of the peak noise. They showed that an interaction process could occur that would result in suppressing the fundamental and thus reducing the peak noise.

The starting point of this analysis is that we consider a turbulent round jet at a sufficiently high speed so that the compressibility is significant. The development of this jet in the unexcited case is assumed to be given by some other means (e.g. analytically or via Reynolds averaged numerical simulations). This jet is then excited by a singlefrequency instability wave. The nonlinear development of this wave will be presented herein based on the integral energy approach. Along with the wave development, the mean flow-spreading rate is also modified and will be presented herein. Since the focus here is the supersonic jet, we must consider the helical modes as they are more amplified 
than the axisymmetric ones. ${ }^{8}$ Once the computations are completed, a description of all the variables of the largestructure disturbance field in and near the jet is given for a particular mode number and frequency. It can be used as the acoustic source to compute the noise radiation field outside of the jet. This is described in a separate paper. ${ }^{9}$

\section{FORMULATION OF THE PROBLEM}

Consider a high-Reynolds number turbulent jet issuing from a nozzle of diameter $D$ in a still air. The jet is shock-free, but the Mach number is high enough for compressibility effects to be significant. The jet is excited by a single-frequency instability wave of Strouhal number $f D / \bar{U}_{j}$. The density and the component velocities are normalized by the jet exit density and axial velocity at the centerline, $\bar{\rho}_{j}$ and $\bar{U}_{j}$, respectively. The pressure is normalized by $\bar{\rho}_{j} \bar{U}_{j}^{2}$, time by $D / \bar{U}_{j}$, and spatial coordinates by $D$. Each flow parameter is split into a time-averaged part $\bar{U}_{i}(x, r, \phi)$, a coherent, wave-like part, $u_{i}^{\prime}(x, r, \phi, t)$, and a fine-scale random turbulence part, $u_{i}^{\prime \prime}(x, r, \phi, t)$. Thus, the velocity can be written :

$$
u_{i}=\bar{U}_{i}(x, r, \phi)+u_{i}^{\prime}(x, r, \phi, t)+u_{i}^{\prime \prime}(x, r, \phi, t)
$$

where $i=1,2,3$. In the cylindrical coordinates, 1 refers to the axial direction $x$ with axial velocity $u, 2$ refers to the radial direction $r$ with radial velocity $v$, and 3 refers to the azimuthal direction $\phi$ with azimuthal velocity $w$. An over bar, $\overline{(\quad)}$, denotes a time-averaged quantity. The pressure and the density are similarly split:

$$
\begin{gathered}
p=\bar{P}(x, r, \phi)+p^{\prime}(x, r, \phi, t)+p^{\prime \prime}(x, r, \phi, t) \\
\rho=\bar{\rho}(x, r, \phi)+\rho^{\prime}(x, r, \phi, t)+\rho^{\prime \prime}(x, r, \phi, t)
\end{gathered}
$$

In the subsequent analysis, however, the random turbulence component will not be explicitly considered any further, thus we have

$$
\begin{aligned}
u_{i} & =\bar{U}_{i}(x, r, \phi)+u_{i}^{\prime}(x, r, \phi, t) \\
p & =\bar{P}(x, r, \phi)+p^{\prime}(x, r, \phi, t) \\
\rho & =\bar{\rho}(x, r, \phi)+\rho^{\prime}(x, r, \phi, t)
\end{aligned}
$$

For the product of the density with the velocities, we get $\left(\rho u_{i}\right)=\left(\bar{\rho}+\rho^{\prime}\right)\left(\bar{U}_{i}+u_{i}^{\prime}\right)=\bar{\rho} \bar{U}_{i}+\bar{\rho} u_{i}^{\prime}+\rho^{\prime} \bar{U}_{i}+\rho^{\prime} u_{i}^{\prime}$

Time-averaging yields

$$
\left(\overline{\rho u_{i}}\right)=\bar{\rho} \bar{U}_{i}+\overline{\rho^{\prime} u_{i}^{\prime}}
$$

and we define

$$
\widetilde{u}_{i} \equiv \rho u_{i}-\overline{\rho u_{i}}=\bar{\rho} u_{i}^{\prime}+\rho^{\prime} \bar{U}_{i}+\rho^{\prime} u_{i}^{\prime}-\overline{\rho^{\prime} u_{i}^{\prime}} .
$$

Neglecting the difference between the product of disturbance variables and the averaged value of the product results in

$$
\widetilde{u}_{i} \approx \bar{\rho} u_{i}^{\prime}+\rho^{\prime} \bar{U}_{i}
$$

\section{Equations of motion}

The formulation begins with the following nondimensionalized continuity and momentum equations in cylindrical coordinates:

$$
\begin{aligned}
\rho_{t} & +(\rho u)_{x}+\frac{1}{r}[r(\rho v)]_{r}+\frac{1}{r}(\rho w)_{\phi}=0 \\
(\rho u)_{t} & +\left(\rho u^{2}+p\right)_{x} \\
& +\frac{1}{r}[r(\rho u v)]_{r}+\frac{1}{r}(\rho u w)_{\phi}=\frac{1}{\operatorname{Re}} \Delta u \\
(\rho v)_{t}+ & (\rho u v)_{x} \\
& +\frac{1}{r}\left[r\left(p+\rho v^{2}\right)\right]_{r}+\frac{1}{r}(\rho v w)_{\phi}-\frac{\rho w^{2}}{r} \\
& =\frac{p}{r}+\frac{1}{\operatorname{Re}}\left[\Delta v-\frac{1}{r^{2}}\left(v+2 w_{\phi}\right)\right] \\
(\rho w)_{t} & +(\rho u w)_{x} \\
& +\frac{1}{r}[r(\rho v w)]_{r}+\frac{1}{r}\left(p+\rho w^{2}\right)_{\phi}+\frac{\rho w v}{r} \\
& =\frac{1}{\operatorname{Re}}\left[\Delta w-\frac{1}{r^{2}}\left(w-2 v_{\phi}\right)\right]
\end{aligned}
$$

where the Reynolds number is $\operatorname{Re}=\bar{\rho}_{j} \bar{U}_{j} D / \mu$. The subscripts denote differentiation and the Laplacian is

$$
\Delta=\frac{\partial^{2}}{\partial x^{2}}+\frac{1}{r} \frac{\partial}{\partial r} r \frac{\partial}{\partial r}+\frac{1}{r^{2}} \frac{\partial^{2}}{\partial \phi^{2}} .
$$

Substituting relations (4) to (8) into (9) to (12), we get:

$$
\begin{aligned}
&\left(\bar{\rho}+\rho^{\prime}\right)_{t}+(\overline{\rho u}+\widetilde{u})_{x} \\
&+\frac{1}{r}[r(\overline{\rho v}+\widetilde{v})]_{r}+\frac{1}{r}(\overline{\rho w}+\widetilde{w})_{\phi}=0 \\
&(\overline{\rho u}+\widetilde{u})_{t}+\left[\bar{P}+p^{\prime}+\left(\bar{U}+u^{\prime}\right)(\overline{\rho u}+\widetilde{u})\right]_{x} \\
&+\frac{1}{r}\left[r\left(\bar{V}+v^{\prime}\right)(\overline{\rho u}+\widetilde{u})\right]_{r}+\frac{1}{r}\left[\left(\bar{W}+w^{\prime}\right)(\overline{\rho u}+\widetilde{u})\right]_{\phi} \\
&=\frac{1}{\operatorname{Re}} \Delta\left(\bar{U}+u^{\prime}\right)
\end{aligned}
$$

$$
\begin{aligned}
& (\overline{\rho v}+\widetilde{v})_{t}+\left[\left(\bar{U}+u^{\prime}\right)(\overline{\rho v}+\widetilde{v})\right]_{x} \\
+ & \frac{1}{r}\left[r\left(\bar{P}+p^{\prime}+\left(\bar{V}+v^{\prime}\right)(\overline{\rho v}+\widetilde{v})\right)\right]_{r} \\
+ & \frac{1}{r}\left[\left(\bar{W}+w^{\prime}\right)(\overline{\rho v}+\widetilde{v})\right]_{\phi}-\frac{1}{r}\left(\bar{W}+w^{\prime}\right)(\overline{\rho w}+\widetilde{w}) \\
= & \frac{1}{r}\left(\bar{P}+p^{\prime}\right) \\
+ & \frac{1}{\operatorname{Re}}\left[\Delta\left(\bar{V}+v^{\prime}\right)-\frac{1}{r^{2}}\left(\bar{V}+v^{\prime}+2\left(\bar{W}+w^{\prime}\right)_{\phi}\right)\right]
\end{aligned}
$$




$$
\begin{aligned}
& (\overline{\rho w}+\widetilde{w})_{t}+\left[\left(\bar{U}+u^{\prime}\right)(\overline{\rho w}+\widetilde{w})\right]_{x} \\
& +\frac{1}{r}\left[r\left(\bar{V}+v^{\prime}\right)(\overline{\rho w}+\widetilde{w})\right]_{r}+\frac{1}{r}\left(\bar{V}+v^{\prime}\right)(\overline{\rho w}+\widetilde{w}) \\
& +\frac{1}{r}\left[\bar{P}+p^{\prime}+\left(\bar{W}+w^{\prime}\right)(\overline{\rho u}+\widetilde{u})\right]_{\phi} \\
& =\frac{1}{\operatorname{Re}}\left[\Delta\left(\bar{W}+w^{\prime}\right)-\frac{1}{r^{2}}\left(\bar{W}+w^{\prime}-2\left(\bar{V}+v^{\prime}\right)_{\phi}\right)\right]
\end{aligned}
$$

These equations will be used to derive a set of equations governing the mean flow and a set of equations governing the large-scale structures.

\section{Mean flow}

The mean flow equations are obtained by time-averaging equations (13) to (16) containing the two-component decomposition. The continuity equation for the mean flow is

$$
(\bar{\rho} \bar{U})_{x}+\frac{1}{r}[r(\bar{\rho} \bar{V})]_{r}+\frac{1}{r}(\bar{\rho} \bar{W})_{\phi}=0
$$

and the three momentum equations for the mean flow are:

$$
\begin{aligned}
& \left(\bar{\rho} \bar{U}^{2}+\overline{u^{\prime} \widetilde{u}}+\bar{P}\right)_{x}+\frac{1}{r}\left[r\left(\bar{\rho} \bar{U} \bar{V}+\overline{v^{\prime} \widetilde{u}}\right)\right]_{r} \\
& +\frac{1}{r}\left(\bar{\rho} \bar{W} \bar{U}+\overline{w^{\prime} \widetilde{u}}\right)_{\phi}=\frac{1}{\operatorname{Re}} \Delta \bar{U} \\
& \left(\bar{\rho} \bar{U} \bar{V}+\overline{u^{\prime} \widetilde{v}}\right)_{x}+\frac{1}{r}\left[r\left(\bar{\rho} \bar{V}^{2}+\overline{v^{\prime} \widetilde{v}}+\bar{P}\right)\right]_{r} \\
& +\frac{1}{r}\left(\bar{\rho} \bar{W} \bar{V}+\overline{w^{\prime} \widetilde{v}}\right)_{\phi}-\frac{1}{r}\left(\bar{\rho} \bar{W}^{2}+\overline{w^{\prime} \widetilde{w}}\right) \\
& =\frac{1}{r} \bar{P}+\frac{1}{\operatorname{Re}}\left[\Delta \bar{V}-\frac{1}{r^{2}}\left(\bar{V}+2 \bar{W}_{\phi}\right)\right] \\
& \left(\bar{\rho} \bar{U} \bar{W}+\overline{u^{\prime} \widetilde{w}}\right)_{x}+\frac{1}{r}\left[r\left(\bar{\rho} \bar{V} \bar{W}+\overline{v^{\prime} \widetilde{w}}\right)\right]_{r} \\
& +\frac{1}{r}\left(\bar{\rho} \bar{W}^{2}+\overline{w^{\prime} \widetilde{w}}+\bar{P}\right)_{\phi}+\frac{1}{r}\left(\bar{\rho} \bar{W} \bar{V}+\overline{v^{\prime} \widetilde{w}}\right) \\
& =\frac{1}{\operatorname{Re}}\left[\Delta \bar{W}-\frac{1}{r^{2}}\left(\bar{W}-2 \bar{V}_{\phi}\right)\right] \text {. }
\end{aligned}
$$

\section{Large-scale structure}

The equations governing the large-scale structures are obtained by subtracting the mean flow equations (17) to (20) from equations (13) to (16). The second order terms are neglected with respect to first order terms and the terms $u_{i}^{\prime} \widetilde{u}_{j}-\overline{u_{i}^{\prime} \widetilde{u}_{j}}$ are also neglected. The continuity and momentum equations for the large-scale structures are:

$$
\begin{gathered}
\rho_{t}^{\prime}+\widetilde{u}_{x}+\frac{1}{r}(r \widetilde{v})_{r}+\frac{1}{r} \widetilde{w}_{\phi}=0 \\
\widetilde{u}_{t}+\left(p^{\prime}+\bar{\rho} \bar{U} u^{\prime}+\widetilde{u} \bar{U}\right)_{x}+\frac{1}{r}\left[r\left(\bar{\rho} \bar{U} v^{\prime}+\widetilde{u} \bar{V}\right)\right]_{r} \\
+\frac{1}{r}\left(\bar{\rho} \bar{U} w^{\prime}+\widetilde{u} \bar{W}\right)_{\phi}=\frac{1}{\operatorname{Re}} \Delta u^{\prime}
\end{gathered}
$$

$$
\begin{aligned}
\widetilde{v}_{t} & +\left(\bar{\rho} \bar{V} u^{\prime}+\widetilde{v} \bar{U}\right)_{x}+\frac{1}{r}\left[r\left(p^{\prime}+\bar{\rho} \bar{V} v^{\prime}+\widetilde{v} \bar{V}\right)\right]_{r} \\
& +\frac{1}{r}\left(\bar{\rho} \bar{V} w^{\prime}+\widetilde{v} \bar{W}\right)_{\phi}-\frac{1}{r}\left(\bar{\rho} \bar{W} w^{\prime}+\widetilde{w} \bar{W}\right) \\
& =\frac{1}{r} p^{\prime}+\frac{1}{\operatorname{Re}}\left[\Delta v^{\prime}-\frac{1}{r^{2}}\left(v^{\prime}+2 w_{\phi}^{\prime}\right)\right]
\end{aligned}
$$

$$
\begin{aligned}
\widetilde{w}_{t} & +\left(\bar{\rho} \bar{W} u^{\prime}+\widetilde{w} \bar{U}\right)_{x}+\frac{1}{r}\left[r\left(\bar{\rho} \bar{W} v^{\prime}+\widetilde{w} \bar{V}\right)\right]_{r} \\
& +\frac{1}{r}\left(p^{\prime}+\bar{\rho} \bar{W} w^{\prime}+\widetilde{w} \bar{W}\right)_{\phi}+\frac{1}{r}\left(\bar{\rho} \bar{W} v^{\prime}+\widetilde{w} \bar{V}\right) \\
& =\frac{1}{\operatorname{Re}}\left[\Delta w^{\prime}-\frac{1}{r^{2}}\left(w^{\prime}-2 v_{\phi}^{\prime}\right)\right]
\end{aligned}
$$

\section{Kinetic energy equations}

Mean flow kinetic energy equation

The mean flow kinetic energy equation is obtained by first multiplying the x-momentum equation (18) by $\bar{U}$, the r-momentum equation (19) by $\bar{V}$, and the $\phi$-momentum equation (20) by $\bar{W}$. Then, the resulting equations are added together. This combined equation is composed of several main terms including the stress terms, the pressure terms, and the viscosity terms. Using the mean flow continuity equation and defining the mean flow kinetic energy as $K=\left(\bar{U}^{2}+\bar{V}^{2}+\bar{W}^{2}\right) / 2$, the combined equation is rearranged to obtain the kinetic energy equation for the mean flow

$$
\begin{aligned}
& \frac{\partial}{\partial x}\left(\bar{\rho} \bar{U} K+\overline{u^{\prime} \widetilde{u}} \bar{U}+\overline{u^{\prime} \widetilde{v}} \bar{V}+\overline{u^{\prime} \widetilde{w}} \bar{W}+\bar{U} \bar{P}\right) \\
& +\frac{1}{r} \frac{\partial}{\partial r}\left[r\left(\bar{\rho} \bar{V} K+\overline{v^{\prime} \widetilde{u}} \bar{U}+\overline{v^{\prime} \widetilde{v}} \bar{V}+\overline{v^{\prime} \widetilde{w}} \bar{W}+\bar{V} \bar{P}\right)\right] \\
& +\frac{1}{r} \frac{\partial}{\partial \phi}\left(\bar{\rho} \bar{W} K+\overline{w^{\prime} \widetilde{u}} \bar{U}+\overline{w^{\prime} \widetilde{v}} \bar{V}+\overline{w^{\prime} \widetilde{w}} \bar{W}+\bar{W} \bar{P}\right) \\
& -\bar{P}\left(\bar{U}_{x}+\frac{1}{r}(r \bar{V})_{r}+\frac{1}{r} \bar{W}_{\phi}\right) \\
& -\bar{U}_{x} \overline{u^{\prime} \widetilde{u}}-\bar{V}_{x} \overline{u^{\prime} \widetilde{v}}-\bar{W}_{x} \overline{u^{\prime} \widetilde{w}}-\bar{U}_{r} \overline{v^{\prime} \widetilde{u}} \\
& -\bar{V}_{r} \overline{v^{\prime} \widetilde{v}}-\bar{W}_{r} \overline{v^{\prime} \widetilde{w}}-\frac{1}{r} \bar{U}_{\phi} \overline{w^{\prime} \widetilde{u}}-\frac{1}{r} \overline{V_{\phi}} \overline{w^{\prime} \widetilde{v}} \\
& -\frac{1}{r} \overline{W_{\phi}} \overline{w^{\prime} \widetilde{w}}-\frac{1}{r} \bar{V} \bar{w}^{\prime} \widetilde{w}+\frac{1}{r} \overline{W^{\prime}} \frac{v^{\prime}}{\operatorname{Re}}\left[\Delta K-\left(\bar{U}_{i x}\right)^{2}-\left(\bar{U}_{i r}\right)^{2}-\frac{1}{r^{2}}\left(\bar{U}_{i \phi}\right)^{2}\right. \\
& =\frac{1}{\operatorname{Re}}\left[\overline{V^{2}}\left(\bar{V}+2 \bar{W}_{\phi}\right)-\frac{\bar{W}_{r^{2}}}{\left.\left(\bar{W}-2 \bar{V}_{\phi}\right)\right]}\right.
\end{aligned}
$$

where $\left(\bar{U}_{i x}\right)^{2}=\left(\bar{U}_{x}\right)^{2}+\left(\bar{V}_{x}\right)^{2}+\left(\bar{W}_{x}\right)^{2}$ and similarly for $\left(\bar{U}_{i r}\right)^{2}$ and $\left(\bar{U}_{i \phi}\right)^{2}$.

\section{Large-scale kinetic energy equation}

The large-scale energy equation is obtained by multiplying the $x$-momentum equation (22) by $u^{\prime}$, the $r$-momentum 
equation (23) by $v^{\prime}$, and the $\phi$-momentum equation (24) by $w^{\prime}$. Again, the resulting equations are added together and the time-average is applied. This equation requires extensive manipulation to obtain its final form. The largescale continuity equation (21) and equation (8) are used to combine terms. Defining the large-scale coherent structure kinetic energy as $Q=\left(u^{\prime 2}+{v^{\prime}}^{2}+{w^{\prime}}^{2}\right) / 2$ and noting that the mean flow is steady, the resulting energy equation for the large-scale structures is

$$
\begin{aligned}
& \frac{\partial}{\partial x}(\bar{\rho} \bar{U} \bar{Q})+\frac{1}{r} \frac{\partial}{\partial r}(r \bar{\rho} \bar{V} \bar{Q})+\frac{1}{r} \frac{\partial}{\partial \phi}(\bar{\rho} \bar{W} \bar{Q}) \\
& +\overline{u^{\prime} p_{x}^{\prime}}+\overline{v^{\prime} p_{r}^{\prime}}+\overline{\frac{w^{\prime} p_{\phi}^{\prime}}{r}}+\overline{u^{\prime} \widetilde{u}} \bar{U}_{x}+\overline{u^{\prime} \widetilde{v}} \bar{U}_{r} \\
& +\frac{\overline{u^{\prime} \widetilde{w}}}{r} \bar{U}_{\phi}+\overline{v^{\prime} \widetilde{u}} \bar{V}_{x}+\overline{v^{\prime} \widetilde{v}} \bar{V}_{r}+\frac{\overline{v^{\prime} \widetilde{w}}}{r} \bar{V}_{\phi}+\overline{w^{\prime} \widetilde{u}} \bar{W}_{x} \\
& +\overline{w^{\prime} \widetilde{v}} \bar{W}_{r}+\frac{\overline{w^{\prime} \widetilde{w}}}{r} \bar{W}_{\phi}+\frac{\overline{w^{\prime} \widetilde{w}}}{r} \bar{V}+\frac{\overline{v^{\prime}}}{r} \bar{W} \\
& =\frac{1}{\operatorname{Re}}\left[\Delta \bar{Q}-\overline{\left(u_{i x}^{\prime}\right)^{2}}-\overline{\left(u_{i r}^{\prime}\right)^{2}}-\frac{\overline{\left(u_{i \phi}^{\prime}\right)^{2}}}{r^{2}}\right. \\
& \left.-\overline{\frac{v^{\prime}}{r^{2}}\left(v^{\prime}+2 w_{\phi}^{\prime}\right)}-\overline{\frac{w^{\prime}}{r^{2}}\left(w^{\prime}-2 v_{\phi}^{\prime}\right)}\right]
\end{aligned}
$$

\section{INTEGRAL FORM OF THE ENERGY EQUATION}

For the round jet, the mean quantities are assumed to be axisymmetric. Thus, $\bar{W}=0$ and $\partial \overline{(~)} / \partial \phi=0$. The energy equations (25) and (26) for the mean flow and for the large-scale structures, respectively, become:

$$
\begin{aligned}
& \frac{\partial}{\partial x}\left(\bar{\rho} \bar{U} K+\overline{u^{\prime} \widetilde{u}} \bar{U}+\overline{u^{\prime} \widetilde{v}} \bar{V}+\bar{U} \bar{P}\right) \\
& +\frac{1}{r} \frac{\partial}{\partial r}\left[r\left(\bar{\rho} \bar{V} K+\overline{v^{\prime} \widetilde{u}} \bar{U}+\overline{v^{\prime} \widetilde{v}} \bar{V}+\bar{V} \bar{P}\right)\right] \\
& -\bar{P}\left(\bar{U}_{x}+\frac{1}{r}(r \bar{V})_{r}\right) \\
& -\bar{U}_{x} \overline{u^{\prime} \widetilde{u}}-\bar{V}_{x} \overline{u^{\prime} \widetilde{v}}-\bar{U}_{r} \overline{v^{\prime} \widetilde{u}}-\bar{V}_{r} \overline{v^{\prime} \widetilde{v}}-\frac{1}{r} \bar{V} \overline{w^{\prime} \widetilde{w}} \\
& =\frac{1}{\operatorname{Re}}\left[\Delta K-\left(\bar{U}_{i x}\right)^{2}-\left(\bar{U}_{i r}\right)^{2}-\frac{\bar{V}^{2}}{r^{2}}\right] \\
& \frac{\partial}{\partial x}(\bar{\rho} \bar{U} \bar{Q})+\frac{1}{r} \frac{\partial}{\partial r}(r \bar{\rho} \bar{V} \bar{Q})+\overline{u^{\prime} p_{x}^{\prime}}+\overline{v^{\prime} p_{r}^{\prime}}+\frac{\overline{w^{\prime} p_{\phi}^{\prime}}}{r} \\
& +\overline{u^{\prime} \widetilde{u}} \bar{U}_{x}+\overline{u^{\prime} \widetilde{v}} \bar{U}_{r}+\overline{v^{\prime} \widetilde{u}} \bar{V}_{x}+\overline{v^{\prime} \widetilde{v}} \bar{V}_{r}+\frac{\overline{w^{\prime} \widetilde{w}}}{r} \bar{V} \\
& =\frac{1}{\operatorname{Re}}\left[\Delta \bar{Q}-\overline{\left(u_{i x}^{\prime}\right)^{2}}-\overline{\left(u_{i r}^{\prime}\right)^{2}}-\frac{\overline{\left(u_{i \phi}^{\prime}\right)^{2}}}{r^{2}}\right. \\
& \left.-\overline{\frac{v^{\prime}}{r^{2}}\left(v^{\prime}+2 w_{\phi}^{\prime}\right)}-\overline{\frac{w^{\prime}}{r^{2}}\left(w^{\prime}-2 v_{\phi}^{\prime}\right)}\right]
\end{aligned}
$$

The usual boundary layer-type approximations are applied to the mean quantities. The radial velocity is much less than the axial velocity, $\bar{V} \ll \bar{U}$, and the axial gradients are much less than the radial gradients, $\partial \overline{(~)} / \partial x \ll \partial \overline{()} / \partial r$. Second order terms are also neglected with respect to first-order terms. Thus, the mean flow energy equation reduces to:

$$
\begin{aligned}
\frac{\partial}{\partial x}\left(\frac{1}{2} \bar{\rho} \bar{U}^{3}\right. & +\bar{U} \bar{P})+\frac{1}{r} \frac{\partial}{\partial r}\left[r\left(\frac{1}{2} \bar{\rho} \bar{V} \bar{U}^{2}+\bar{V} \bar{P}\right)\right] \\
& -\bar{P}\left(\bar{U}_{x}+\frac{1}{r}(r \bar{V})_{r}\right)-\bar{U}_{r} \overline{v^{\prime} \widetilde{u}} \\
& =\frac{1}{\operatorname{Re}}\left[\frac{1}{r}\left(r\left(\frac{\bar{U}^{2}}{2}\right)_{r}\right)_{r}-\left(\bar{U}_{r}\right)^{2}\right]
\end{aligned}
$$

Multiplying equation (29) by $r$ and integrating over $r$, we get:

$$
\begin{aligned}
& \frac{d}{d x} \int_{0}^{\infty}\left(\frac{1}{2} \bar{\rho} \bar{U}^{3}+\bar{U} \bar{P}\right) r d r+[r \bar{V} \bar{P}]_{r=0}^{r \rightarrow \infty} \\
& -\int_{0}^{\infty} \bar{P} \bar{U}_{x} r d r-\int_{0}^{\infty} \bar{P}(r \bar{V})_{r} d r \\
& -\int_{0}^{\infty} \bar{U}_{r} \overline{v^{\prime} \widetilde{u}} r d r=-\frac{1}{\operatorname{Re}} \int_{0}^{\infty}\left(\bar{U}_{r}\right)^{2} r d r
\end{aligned}
$$

since:

$$
\left[r \bar{\rho} \bar{V} \frac{\bar{U}^{2}}{2}\right]_{r=0}^{r \rightarrow \infty}=0 \quad \text { and } \quad\left[r\left(\frac{\bar{U}^{2}}{2}\right)\right]_{r=0}^{r \rightarrow \infty}=0
$$

The mean flow pressure is assumed to be constant across the jet. The integral form of the mean energy equation reduces to the following simple form.

$\frac{d}{d x} \int_{0}^{\infty} \frac{1}{2} \bar{\rho} \bar{U}^{3} r d r=\int_{0}^{\infty} \bar{U}_{r} \overline{v^{\prime} \widetilde{u}} r d r-\frac{1}{\operatorname{Re}} \int_{0}^{\infty}\left(\bar{U}_{r}\right)^{2} r d r$

Similarly, the energy equation for the coherent component reduces to:

$$
\begin{aligned}
& \frac{\partial}{\partial x}(\bar{\rho} \bar{U} \bar{Q})+\frac{1}{r} \frac{\partial}{\partial r}(r \bar{\rho} \bar{V} \bar{Q}) \\
& +\overline{u^{\prime} p_{x}^{\prime}}+\overline{v^{\prime} p_{r}^{\prime}}+\frac{\overline{w^{\prime} p_{\phi}^{\prime}}}{r}+\overline{u^{\prime} \widetilde{v}} \bar{U}_{r} \\
& =\frac{1}{\operatorname{Re}}\left[\Delta \bar{Q}-\overline{\left(u_{i x}^{\prime}\right)^{2}}-\overline{\left(u_{i r}^{\prime}\right)^{2}}-\frac{\overline{\left(u_{i \phi}^{\prime}\right)^{2}}}{r^{2}}\right. \\
& \left.-\overline{\frac{v^{\prime}}{r^{2}}\left(v^{\prime}+2 w_{\phi}^{\prime}\right)}-\overline{\frac{w^{\prime}}{r^{2}}\left(w^{\prime}-2 v_{\phi}^{\prime}\right)}\right]
\end{aligned}
$$

Multiplying (31) by $r$ and integrating over $r$, we get:

$$
\frac{d}{d x} \int_{0}^{\infty}(\bar{\rho} \bar{U} \bar{Q}) r d r=
$$




$$
\begin{aligned}
& -\int_{0}^{\infty}\left(\overline{u^{\prime} p_{x}^{\prime}}+\overline{v^{\prime} p_{r}^{\prime}}+\frac{\overline{w^{\prime} p_{\phi}^{\prime}}}{r}\right) r d r-\int_{0}^{\infty} \overline{u^{\prime} \widetilde{v}} \bar{U}_{r} r d r \\
& -\frac{1}{\operatorname{Re}} \int_{0}^{\infty}\left[\overline{\left(u_{i x}^{\prime}\right)^{2}}+\overline{\left(u_{i r}^{\prime}\right)^{2}}+\frac{\overline{\left(u_{i \phi}^{\prime}\right)^{2}}}{r^{2}}\right. \\
& \left.+\overline{\frac{v^{\prime}}{r^{2}}\left(v^{\prime}+2 w_{\phi}^{\prime}\right)}+\overline{\frac{w^{\prime}}{r^{2}}\left(w^{\prime}-2 v_{\phi}^{\prime}\right)}\right] r d r
\end{aligned}
$$

since

$$
\left[r \bar{\rho} \bar{V} \bar{Q}_{r=0}^{r \rightarrow \infty}=0 \quad \text { and } \quad\left[r \bar{Q}_{r}\right]_{r=0}^{r \rightarrow \infty}=0 .\right.
$$

The physical interpretation of the terms appearing in the energy equations is obvious. For the mean flow equation, the left side is the mean flow advection of the mean flow kinetic energy. The first term on the right-hand side of (30) is the energy transfer from the mean flow to the coherent structure, and the second term is the viscous dissipation of the mean flow energy. As for the coherent mode equation (32); the left-hand side is the mean flow advection of the coherent mode kinetic energy. The first term in the righthand side is the work done by the coherent mode pressure gradients, the second term is the energy transfer from the mean flow to the coherent mode, and the last term is the coherent mode energy dissipation.

\section{SHAPE ASSUMPTIONS}

To solve the above system of two energy equations, shape assumptions need to be made regarding the radial profiles. The mean flow is assumed to be a known function of $r$ and $\theta$, where $\theta$ is the momentum thickness of the jet shear layer, and the coherent structure profiles are assumed to follow that of the locally-parallel, linear stability theory.

\section{Single azimuthal modes}

Considering single-frequency, single-azimuthal number modes, the normal mode composition suggests that these modes can be represented as:

$$
\begin{aligned}
u_{i}^{\prime}(x, r, \phi, t) & =\hat{u}_{i}(r) A(x) \exp (\Psi+i n \phi)+c c \\
p^{\prime}(x, r, \phi, t) & =\hat{p}(r) A(x) \exp (\Psi+i n \phi)+c c \\
\rho^{\prime}(x, r, \phi, t) & =\hat{\rho}(r) A(x) \exp (\Psi+i n \phi)+c c \\
\Psi & =i \int_{0}^{x} \alpha d \chi-i \omega t
\end{aligned}
$$

where $\left({ }^{\wedge}\right)$ denotes the radial shape function of the transverse coordinate $r$ at a given location along the jet. $\hat{u}_{i}(r)$, $\hat{p}(r)$, and $\hat{\rho}(r)$ are eigenfunctions corresponding to a given $n$ and $\omega$. Here, $n$ is the azimuthal wave number indicating the rotation around the jet centerline, $\alpha$ is the wave number and $c c$ denotes the complex conjugate. $A(x)$ is the complex amplitude function of $x$ and is to be determined by a nonlinear analysis. In the nonlinear analysis, the linear growth rate as determined by the imaginary part of $\alpha$ is absorbed into $A(x)$. The radial shape functions are normalized such that

$$
\int_{0}^{\infty}\left(|\hat{u}|^{2}+|\hat{v}|^{2}+|\hat{w}|^{2}\right) r d r=1
$$

Substituting the mode definitions into equation (30) and extending the definition of the averaging process to include the azimuthal direction,

$$
\overline{(\quad)}=\lim _{T \rightarrow \infty} \frac{1}{2 \pi T} \int_{0}^{2 \pi} \int_{0}^{T}(\quad) d t d \phi,
$$

where $T$ is a large time scale, we obtain

$$
\frac{d \theta}{d x} \frac{d I_{a m}}{d \theta}=|A|^{2}\left(I_{m w 1}+I_{m w 2}\right)-\frac{1}{\mathrm{Re}} I_{m d}
$$

where

$$
\begin{aligned}
I_{a m} & =\frac{1}{2} \int_{0}^{\infty} \bar{\rho} \bar{U}^{3} r d r \\
I_{m w 1} & =\int_{0}^{\infty}\left(\hat{v} \hat{u}^{*}+c c\right) \bar{\rho} \frac{\partial \bar{U}}{\partial r} r d r \\
I_{m w 2} & =\int_{0}^{\infty}\left(\hat{v} \hat{\rho}^{*}+c c\right) \bar{U} \frac{\partial \bar{U}}{\partial r} r d r \\
I_{m d} & =\int_{0}^{\infty}\left(\frac{\partial \bar{U}}{\partial r}\right)^{2} r d r
\end{aligned}
$$

In this analysis, the mean flow is characterized by the momentum thickness rather than by the axial distance. Hence, the integrals in the energy equations are dependent on $\theta$ instead of $x$.

Upon substitution into equation (32), we obtain for the coherent mode:

$$
\frac{d\left(I_{a w}|A|^{2}\right)}{d x}=|A|^{2}\left(-I_{m w 1}-I_{m w 3}-I_{p}-\frac{1}{\operatorname{Re}} I_{w d}\right)
$$

where

$$
\begin{aligned}
I_{a w}= & \int_{0}^{\infty}\left(|\hat{u}|^{2}+|\hat{v}|^{2}+|\hat{w}|^{2}\right) \bar{\rho} \bar{U} r d r \\
I_{m w 3}= & \int_{0}^{\infty}\left(\hat{u} \hat{\rho}^{*}+c c\right) \bar{V} \frac{\partial \bar{U}}{\partial r} r d r \\
I_{p}= & \int_{0}^{\infty}\left[\hat{u}(i \alpha \hat{p})^{*}+\hat{v} \frac{\partial \hat{p}^{*}}{\partial r}+\frac{\hat{w}}{r}(i n \hat{p})^{*}+c c\right] r d r \\
I_{w d}= & 2 \int_{0}^{\infty}\left[\left(|\alpha|^{2}+\frac{n^{2}}{r^{2}}\right)\left(|\hat{u}|^{2}+|\hat{v}|^{2}+|\hat{w}|^{2}\right)\right. \\
& +\frac{1}{r^{2}}\left(|\hat{v}|^{2}+|\hat{w}|^{2}\right)-\frac{2}{r^{2}}\left(i n \hat{v} \hat{w}^{*}+c c\right) \\
& \left.+\left(\left|\frac{\partial \hat{u}}{\partial r}\right|^{2}+\left|\frac{\partial \hat{v}}{\partial r}\right|^{2}+\left|\frac{\partial \hat{w}}{\partial r}\right|^{2}\right)\right] r d r
\end{aligned}
$$




\section{Pair of oblique waves}

Experimental observations have indicated that the helical modes appear in the form of a symmetrical pair with $n$, the mode number, set to both $\pm 1 .^{8}$ In this case, the normal mode definitions are modified as follows:

$$
\begin{aligned}
u^{\prime}(x, r, \phi, t) & =\hat{u}(r) A(x) \exp (\Psi) \cos (n \phi)+c c \\
v^{\prime}(x, r, \phi, t) & =\hat{v}(r) A(x) \exp (\Psi) \cos (n \phi)+c c \\
w^{\prime}(x, r, \phi, t) & =\hat{w}(r) A(x) \exp (\Psi) \sin (n \phi)+c c \\
p^{\prime}(x, r, \phi, t) & =\hat{p}(r) A(x) \exp (\Psi) \cos (n \phi)+c c \\
\rho^{\prime}(x, r, \phi, t) & =\hat{\rho}(r) A(x) \exp (\Psi) \cos (n \phi)+c c
\end{aligned}
$$

When these definitions are substituted into the integral equations (30) and (32) and the averaging process is applied, we obtain the following sets of equations.

$$
\frac{d \theta}{d x} \frac{d I_{a m}}{d \theta}=|A|^{2}\left(I_{m w 1}+I_{m w 2}\right)-\frac{1}{\mathrm{Re}} I_{m d}
$$

where

$$
\begin{aligned}
& I_{a m}=\frac{1}{2} \int_{0}^{\infty} \bar{\rho} \bar{U}^{3} r d r \\
& I_{m w 1}=\frac{1}{2} \int_{0}^{\infty}\left(\hat{v} \hat{u}^{*}+c c\right) \bar{\rho} \frac{\partial \bar{U}}{\partial r} r d r \\
& I_{m w 2}=\frac{1}{2} \int_{0}^{\infty}\left(\hat{v} \hat{\rho}^{*}+c c\right) \bar{U} \frac{\partial \bar{U}}{\partial r} r d r \\
& I_{m d}=\int_{0}^{\infty}\left(\frac{\partial \bar{U}}{\partial r}\right)^{2} r d r \\
& \frac{d\left(I_{a w}|A|^{2}\right)}{d x}=|A|^{2}\left(-I_{m w 1}-I_{m w 3}-I_{p}-\frac{1}{\operatorname{Re}} I_{w d}\right)
\end{aligned}
$$

where

$$
\begin{aligned}
I_{a w}= & \frac{1}{2} \int_{0}^{\infty}\left(|\hat{u}|^{2}+|\hat{v}|^{2}+|\hat{w}|^{2}\right) \bar{\rho} \bar{U} r d r \\
I_{m w 3}= & \frac{1}{2} \int_{0}^{\infty}\left(\hat{u} \hat{\rho}^{*}+c c\right) \bar{V} \frac{\partial \bar{U}}{\partial r} r d r \\
I_{p}= & \frac{1}{2} \int_{0}^{\infty}\left[\hat{u}(i \alpha \hat{p})^{*}+\hat{v} \frac{\partial \hat{p}^{*}}{\partial r}-\frac{n \hat{w} \hat{p}^{*}}{r}+c c\right] r d r \\
I_{w d}= & \int_{0}^{\infty}\left[\left(|\alpha|^{2}+\frac{n^{2}}{r^{2}}\right)\left(|\hat{u}|^{2}+|\hat{v}|^{2}+|\hat{w}|^{2}\right)\right. \\
& +\frac{1}{r^{2}}\left(|\hat{v}|^{2}+|\hat{w}|^{2}\right)+\frac{2 n}{r^{2}}\left(\hat{v} \hat{w}^{*}+c c\right) \\
& \left.+\left(\left|\frac{\partial \hat{u}}{\partial r}\right|^{2}+\left|\frac{\partial \hat{v}}{\partial r}\right|^{2}+\left|\frac{\partial \hat{w}}{\partial r}\right|^{2}\right)\right] r d r
\end{aligned}
$$

The radial shape functions are normalized such that

$$
\int_{0}^{\infty}\left(|\hat{u}|^{2}+|\hat{v}|^{2}+|\hat{w}|^{2}\right) r d r=\left\{\begin{array}{l}
1, n=0 \\
2, n>0
\end{array}\right.
$$

For the incompressible case, the above equations reduce to that of Lee \& Liu. ${ }^{10}$

\section{Mean flow}

In previous studies using the energy integral technique, the shape assumption for the mean flow was provided by analytic equations describing the velocity and density profiles. ${ }^{1,3,10}$ This works reasonably well in the initial potential core region of the jet where the shear layer is well described by a hyperbolic tangent or other functions and the locally-parallel flow instability wave is predominantly growing. In order to describe the full axial development of growth and decay of the instability wave, the mean flow must not only be described through the potential core region, but also possibly downstream into the fully developed flow region. An analytic function description of the mean flow would not be smoothly continuous throughout this domain. Furthermore, an analytic function description of the mean axial velocity (e. g. hyperbolic tangent) combined with the Crocco-Busemann description of the density profile provides a nonphysical radial velocity profile. A proper physical description for the radial velocity profile is required for computing integral $I_{m w 3}$. These objections are overcome by determining the mean flow numerically. We compute the mean flow using the procedure of Dahl \& Morris ${ }^{11}$ with the addition that the radial velocity profile is computed from the mean continuity equation (17). Thus, the numerical calculations provide the mean flow profile shapes that smoothly transition from the initial region to the fully developed region and that can be parameterized by $\theta$ as required by the analysis.

The computational scheme for the mean flow of a jet was developed using a set of compressible, Reynolds-averaged, boundary layer equations with a modified mixing length model to determine the Reynolds stresses. In nondimensional form, the Reynolds stresses were computed by

$$
-\bar{\rho} \overline{u^{\prime} v^{\prime}}=\frac{\mu_{\mathrm{T}}}{\bar{\rho}_{j} \bar{U}_{j} D} \frac{\partial \bar{U}}{\partial r}
$$

where $\mu_{\mathrm{T}}$ is a turbulent viscosity and is a function of $r$ and $x$. Here, the unsteady terms represent all scales of turbulence. If the fluid viscosity is given by $\mu$, then in the shear layer $\mu_{\mathrm{T}} \gg \mu$.

\section{Linear stability}

The coherent structure profiles are determined from a locally-parallel, linear stability theory. Given the known complications that arise when using inviscid theory to compute eigenvalues and eigenfunctions of the damped portions of the instability wave, a compressible, viscous approach was used for the stability calculations following the work of Morris. ${ }^{12,13}$ The compressible equations of motion are linearized about a locally-parallel flow and the compressible parts of the viscous terms in the momentum equations are neglected. In the energy equation, both viscosity and diffusion effects are neglected. After applying the modal decomposition for single azimuthal modes, the 
governing linearized equations become

$$
\begin{gathered}
\frac{\partial \hat{v}}{\partial r}+\frac{\hat{v}}{r}+\frac{i n}{r} \hat{w}+i \alpha \hat{u}-i M_{j}^{2}(\omega-\alpha \bar{U}) \hat{p}=0 \\
\frac{\partial^{2} \hat{v}}{\partial r^{2}}+\frac{1}{r} \frac{\partial \hat{v}}{\partial r}-\left[\lambda^{2}+\frac{n^{2}+1}{r^{2}}\right] \hat{v}-\frac{i 2 n}{r^{2}} \hat{w}-\operatorname{Re} \frac{\partial \hat{p}}{\partial r}=0 \\
\frac{\partial^{2} \hat{w}}{\partial r^{2}}+\frac{1}{r} \frac{\partial \hat{w}}{\partial r}-\left[\lambda^{2}+\frac{n^{2}+1}{r^{2}}\right] \hat{w}+\frac{i 2 n}{r^{2}} \hat{v}-\frac{i n \operatorname{Re}}{r} \hat{p}=0 \\
\frac{\partial^{2} \hat{u}}{\partial r^{2}}+\frac{1}{r} \frac{\partial \hat{u}}{\partial r}-\left[\lambda^{2}+\frac{n^{2}}{r^{2}}\right] \hat{u}-\bar{\rho} \frac{\partial \bar{U}}{\partial r} \hat{v}-i \alpha \operatorname{Re} \hat{p}=0
\end{gathered}
$$

where $\lambda^{2}=\alpha^{2}-i \bar{\rho} \operatorname{Re}(\omega-\alpha \bar{U})$. For a pair of oblique waves, the linearized equations are the same except the $\hat{w}$ is replaced by $-i \hat{w}$ in equations (46).

Using the boundary conditions given by Morris, ${ }^{13}$ we obtain an eigenvalue problem for $\alpha$. We used a finitedifference approximation to discretize the system of equations (46). The eigenvalue was found from diagonalizing the resulting matrix using a Newton iteration for refinement. The four eigenfunctions in these equations were then found using the inverse power method and normalizations given previously. The density disturbance eigenfunction was obtained from the continuity equation.

$$
\hat{\rho}=\frac{-i \hat{v}}{(\omega-\alpha \bar{U})} \frac{\partial \bar{\rho}}{\partial r}+\bar{\rho} M_{j}^{2} \hat{p}
$$

The eigenvalue depends not only on the flow profiles, but it also depends on the parameters $n, M_{j}, \omega$, and Re. The mode number, the Mach number, and the frequency are easily chosen parameters of interest. The choice of Reynolds number is not as certain. Morris ${ }^{13}$ has shown how the eigenvalue can depend on the choice of Re. In calculations of viscous stability, setting Re to a constant has been typical. ${ }^{14}$ We propose here that since the eigenvalues are computed based on local flow conditions that a local Reynolds number be used, also. Using the numerical mean flow calculations, we determine a local Re from

$$
\operatorname{Re}=\frac{\bar{\rho}_{j} \bar{U}_{j} D}{\left(\mu_{\mathrm{T}}\right)_{\max }}
$$

based on equation (45). This represents the maximum effect that turbulent viscosity has on the large structures.

Though this approach to local stability includes viscous effects, it is found to have the same limitations as linear, inviscid theory. ${ }^{15}$ The analytically continuous eigenvalue solution for a damped, supersonically traveling mode has corresponding eigenfunctions that diverge for large $r$ rather than converging as required to compute the integrals $I_{p}$ and $I_{w d}$. This limits the Mach number for the application of this integral technique.

\section{Mean flow}

\section{NUMERICAL RESULTS}

The calculations were performed for seven jets with exit Mach numbers ranging from 0.3 to 2.1 in increments of

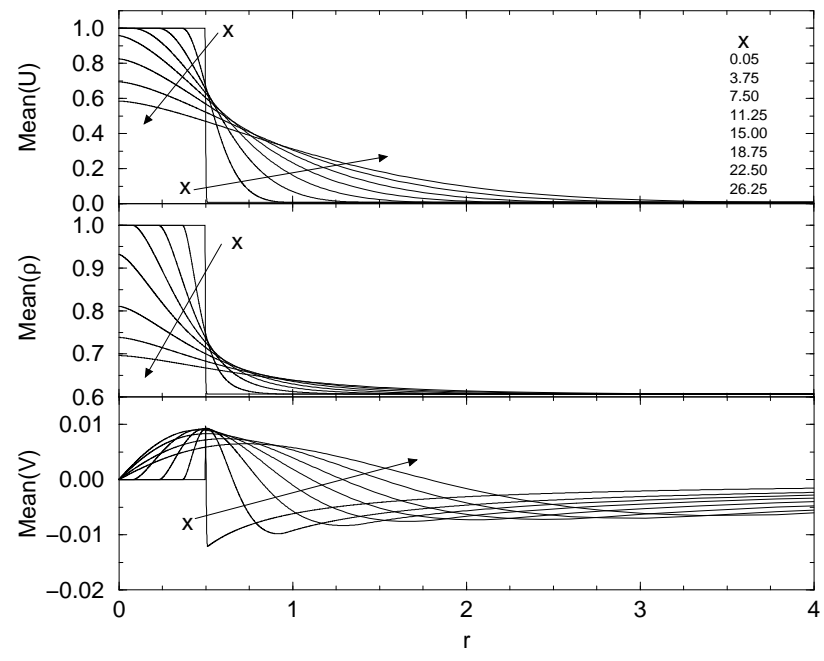

Fig. 1 Jet mean flow profiles computed at 8 axial locations. $M_{j}=1.8 ; T_{o}=T_{\infty}$.

0.3. In all cases, the jets were cold with the jet exit total temperature equal to the ambient temperature. As the flow becomes supersonic, the jets were perfectly expanded. An example of the computed jet profiles is shown in Figure 1 for a Mach 1.8 cold jet. The mean flow profiles have smooth gradients in the radial direction and smoothly transition from the potential core region to the fully developed region downstream. The radial velocity exhibits physically realistic behavior. The flow is outward, a positive velocity, toward the shear layer from the high speed potential core and inward, a negative velocity, outside of the jet indicating entrainment of the outer fluid. These flow calculations meet the requirements indicated for the stability calculations.

The mean flow calculations also provide the local momentum thickness and the local Reynolds number required for the stability and the energy integral calculations. The momentum thickness, $\theta$, is computed based on only the velocity profile and the local Reynolds number is computed from equation (48) during the mean flow computations as part of the turbulence model. The results for the seven jets is shown in Figure 2. The jet spreading rate decreases with Mach number, as expected, with the momentum thickness remaining about the same, $\theta \approx 0.22$, at the end of the potential core as indicated for the $M_{j}=0.3$ and the $M_{j}=1.8$ jet cases. The local Reynolds number decreases rapidly through the potential core from its initial value at the jet exit.

\section{Local stability}

Using the mean flow as input, the local stability characteristics were computed for each jet. The eigenvalue or local wave number was determined from equations (46) where the pair of oblique waves form was used in the computations. Results are shown in Figure 3 for each jet where 

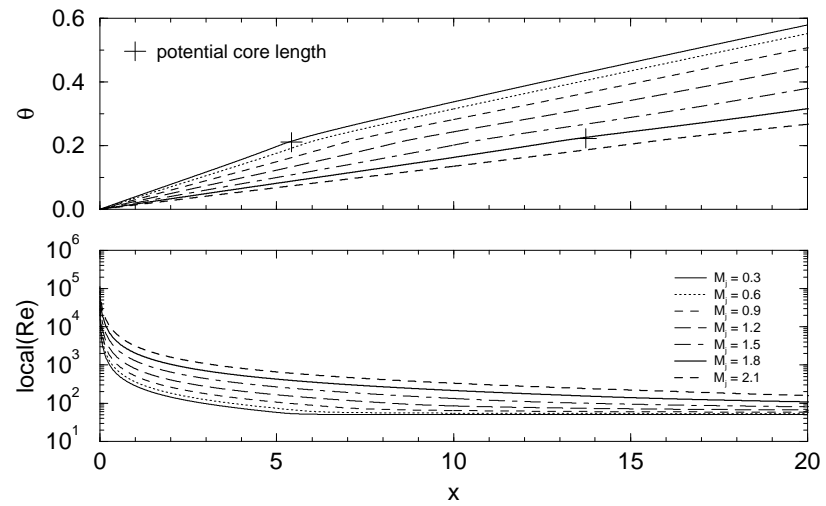

Fig. 2 Axial variation of local momentum thickness, $\theta$, and local Reynolds number, Eq. (48), computed for 7 jet Mach numbers.

the Strouhal number was set to 0.2 and the azimuthal mode number was $n= \pm 1$. The local wave number, $\alpha$, is complex consisting of a real part, $\alpha_{r}$, that governs the axial phase change of the instability wave and an imaginary part, $\alpha_{i}$, that relates to the local growth or decay of the wave. In this figure, a negative $\alpha_{i}$ represents a growing mode and a positive value represents a decaying mode. We can see that the growth rate decreases with an increase in Mach number. This is expected; but, the decrease in growth rate is also a function of the local decrease in Reynolds number. ${ }^{13}$

The local phase velocity is computed from $\omega / \alpha_{r}$. In Figure 3 , it is shown relative to the ambient speed of sound. The relative phase velocity increases directly as the Mach number increases. It is an indication of the ability of the instability wave to directly radiate noise as the phase velocity becomes supersonic relative to the ambient speed of sound. Low speed jets do not radiate sound from this type of source. As the jet speed increases, the higher phase velocity combined with the growth and decay behavior of the instability wave contribute to the ability of these waves to radiate noise. ${ }^{15}$

\section{Local energy integrals}

The four integral terms in equation (43) govern the advection, transfer, and dissipation of the mean kinetic energy. The results of the computations of these integrals for the seven jets are shown in Figure 4. As a function of $\theta$, the advection of the mean flow kinetic energy, as represented by the integral $I_{a m}$, is essentially the same for these jets. This indicates that the integral is related to the jet spreading rate. The rate of change of $I_{a m}$ is initially constant and then gradually decreases. The energy moves downstream as the jet spreads and then downstream of the potential core, the advection slows.

The next two integrals, $I_{m w 1}$ and $I_{m w 2}$, represent the transfer of energy from the mean flow to the coherent structures. The integral $I_{m w 1}$ has the classical form for the velocity shear driven generation of the large-scale structures.
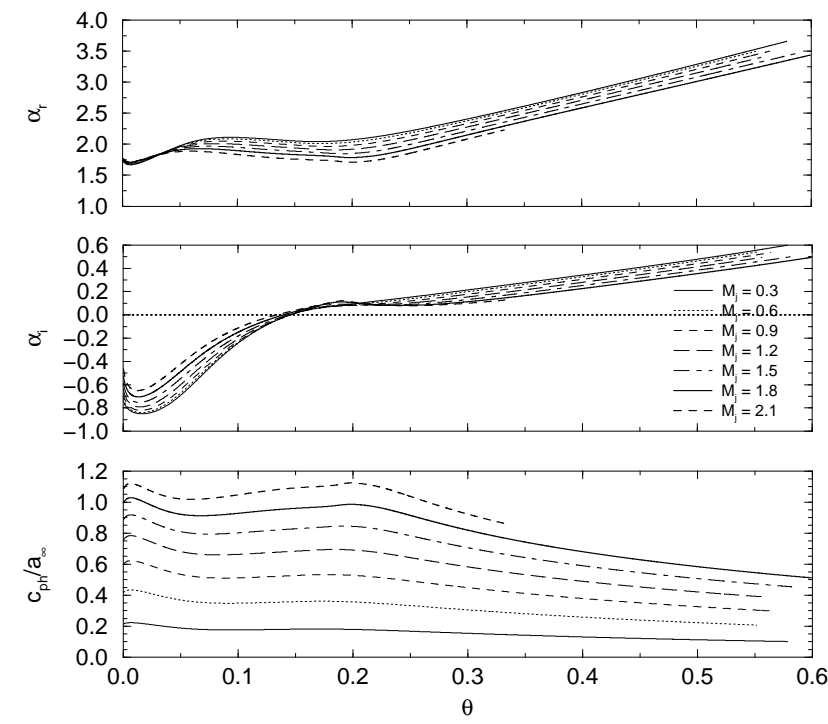

Fig. 3 Local wave number $\alpha=\alpha_{\mathbf{r}}+\mathbf{i} \alpha_{\mathbf{i}}$ and phase velocity, $\mathbf{c}_{\mathbf{p h}}=\omega / \alpha_{\mathbf{r}}$, as a function of jet momentum thickness. $\mathbf{n}= \pm \mathbf{1} ; \mathrm{fD} / \overline{\mathrm{U}}_{\mathrm{j}}=\mathbf{0 . 2}$.

It is negative indicating energy transfer from the mean flow to the modes. Its magnitude is larger for low speed jets and decreases in magnitude as the Mach number increases becoming a less efficient energy transfer process. However, this view has to be balanced by the presence of $I_{m w 2}$. This second integral is present due to compressibility effects. At low speed, the magnitude of $I_{m w 2}$ is negligible. Its effect steadily increases as the Mach number increases until at $M_{j}=2.1$, for example, $I_{m w 1}$ and $I_{m w 2}$ have similar magnitude. Part of the reason for the increased magnitude of $I_{m w 2}$ can be seen by explicitly substituting for $\hat{\rho}$ in the integral using equation (47). The shear of the density profile is a dominant factor in determining the magnitude of $I_{m w 2}$. As the Mach number increased, the jet density increased relative to ambient conditions increasing the density shear rate.

One computational difficulty created with the current viscous stability method is the presence of the pole in $\hat{\rho}$ as the imaginary part of $\alpha$ passes from negative to positive (Eq. (47)). This must be accounted for in the integrals containing $\hat{\rho}$ along with the subsequent branch cut associated with damped modes. The discontinuities present in the plots of $I_{m w 2}$ are clear evidence of the problem.

The final integral, $I_{m d}$, concerns viscous dissipation of the mean kinetic energy. In combination with the local Reynolds number, it is initially two orders of magnitude less than the other integrals and only becomes effective when the energy transfer integrals approach zero. The dissipation is about constant in the potential core region and then decreases downstream. There is more dissipation for lower speed jets than for the higher speed jets.

The next set of integrals, shown in Figure 5, govern the 

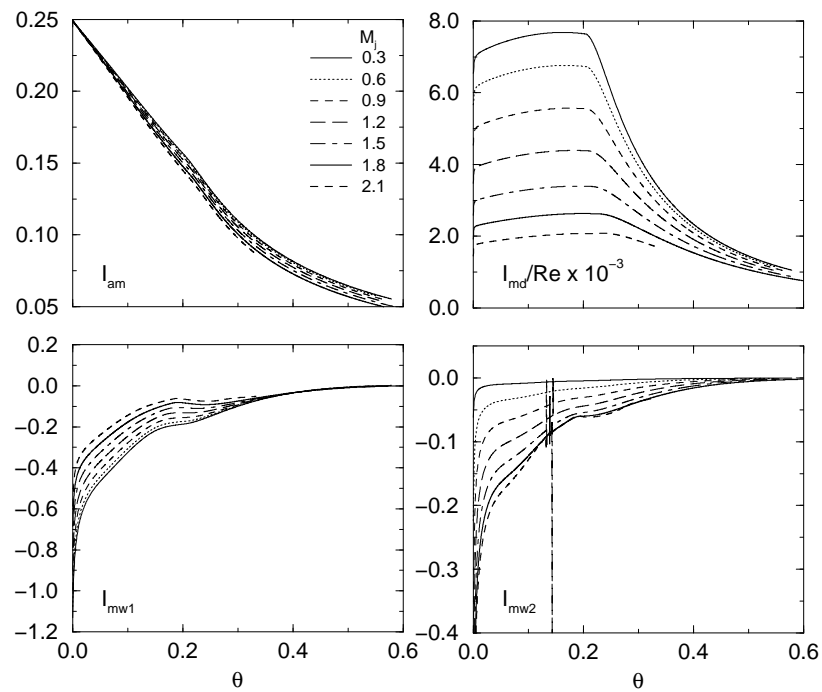

Fig. 4 Energy integrals from the mean flow energy equation (43). $n= \pm 1 ; f D / \bar{U}_{j}=0.2$.
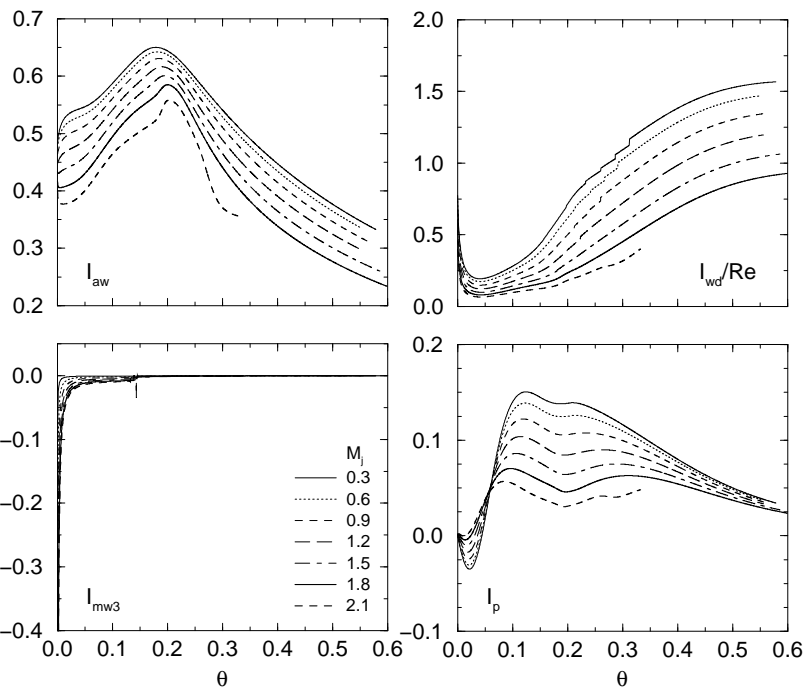

Fig. 5 Energy integrals from the coherent structure energy equation (44). $n= \pm 1 ; \mathrm{fD} / \overline{\mathrm{U}}_{\mathrm{j}}=0.2$.

kinetic energy in the coherent modes; in this case, a pair of oblique modes. The energy comes from the mean flow through $I_{m w 1}$ which now is positive. As it decreases in magnitude, the work done by the coherent mode pressure gradients, $I_{p}$, and the dissipation term begin to reduce the energy in the coherent mode. Soon, the dissipation quickly dominates to rapidly decrease the mode energy. The effect of the compressibility integral $I_{m w 3}$ has some initial effects of adding energy to the coherent modes, but it quickly has negligible effects on further energy transfers.

\section{Nonlinear calculations}

Once the energy integrals were computed, the instability wave amplitude function was determined from the solu-

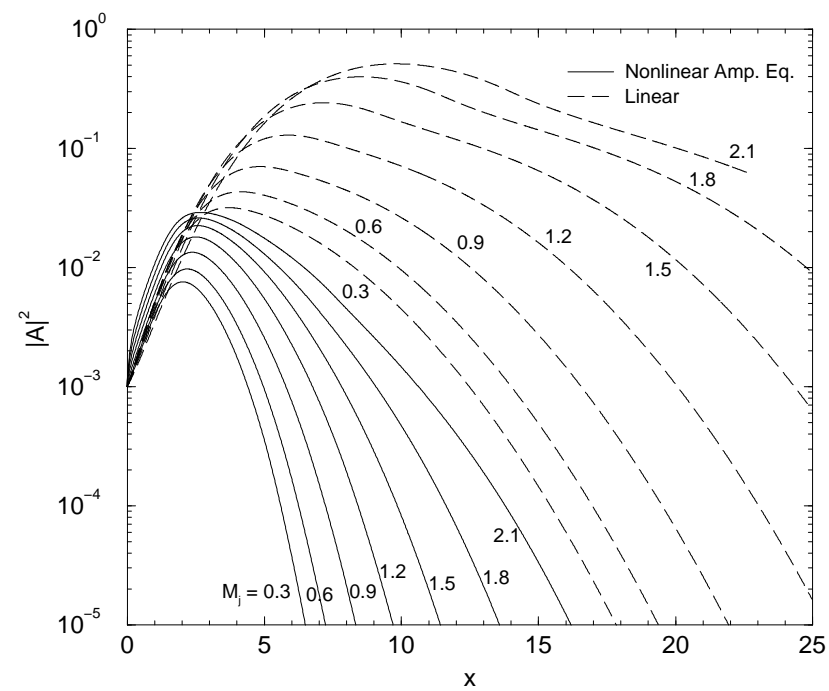

Fig. 6 Amplitude function magnitude comparisons between nonlinear and linear calculations. $\mathrm{n}= \pm \mathbf{1} ; \mathrm{fD} / \overline{\mathrm{U}}_{\mathrm{j}}=\mathbf{0 . 2}$; $\left|A_{o}\right|^{2}=10^{-3}$.

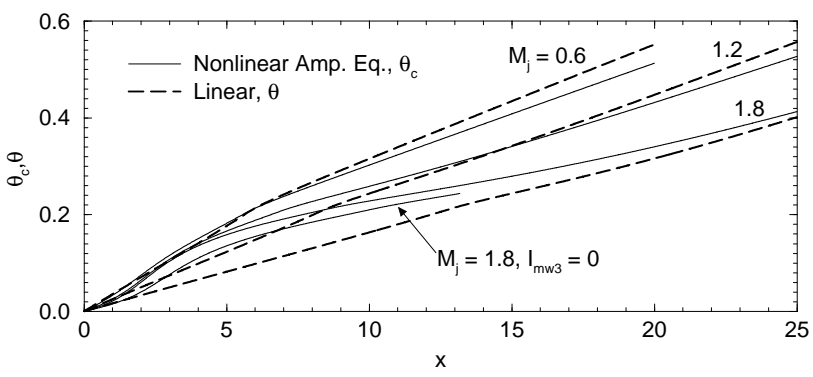

Fig. 7 Shear layer growth comparisons for nonlinear calculations. $\mathrm{n}= \pm 1 ; \mathrm{fD} / \overline{\mathrm{U}}_{\mathrm{j}}=0.2 ;\left|\mathrm{A}_{\mathrm{o}}\right|^{2}=10^{-3}$.

tion of the nonlinear differential equations (43) and (44). An initial value differential equation solver implementing a degree-1 Taylor series scheme with fixed point iteration was used. ${ }^{16}$ The energy integral results were interpolated as necessary to meet the requirements of the scheme. Only the choice of the initial amplitude was needed to start the integration at $x=0$, where $\theta$ was given by the flow calculations.

The results for the computed amplitude function magnitudes for the seven jets are shown in Figure 6 . The Strouhal number was set to 0.2 , the mode number was $n= \pm 1$, and the initial amplitude $\left|A_{o}\right|^{2}$ was $10^{-3}$. The solid lines represent the nonlinear development of the amplitude function. The dashed lines are the corresponding linear instability wave amplitude functions determined from $\left|A_{o}\right| \exp \left(-\int_{0}^{x} \alpha_{i} d \chi\right)$. It can be seen that in this case the nonlinear amplitude reaches a peak and begins to decay much sooner than the linear amplitude. This effect becomes more pronounced as the Mach number increases. At this level of initial amplitude, the mode energy causes increased jet spreading that is more pronounced as Mach 


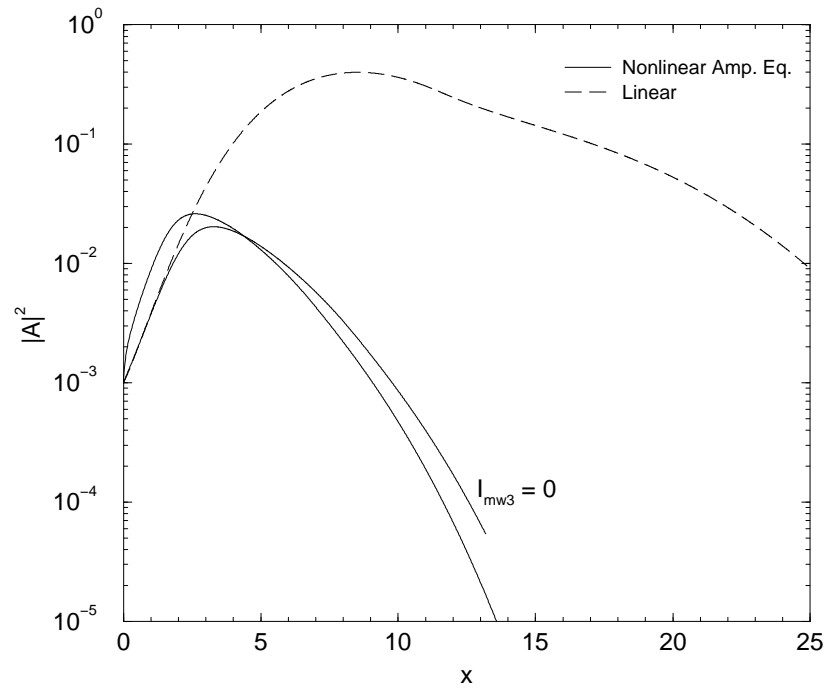

Fig. 8 Amplitude function magnitude comparison with and without the coherent structure compressibility effects integral $I_{\text {mw3 }} . M_{j}=1.8 ; n= \pm 1 ; f D / \bar{U}_{j}=0.2 ;\left|A_{o}\right|^{2}=10^{-3}$.

number increases as shown in Figure 7 for three example jet Mach numbers. The increased jet spreading conversely lessens energy transfer into the mode leading to earlier saturation and decay for higher Mach number nonlinear amplitudes compared to linear amplitudes.

It is interesting to notice that the initial small nonlinear amplitude does not coincide with the linear amplitude. Intuitively, the expectation would be that nonlinear behavior would begin to take affect after a period of linear growth. An examination of the energy exchange integrals is revealing. As mentioned earlier, the integral $I_{m w 3}$ has the effect of initially adding mean flow energy into the coherent modes. It contains terms with the radial mean velocity, $\bar{V}$, and the mean density shear, $\partial \bar{\rho} / \partial r$, which means that $I_{m w 3}$ would not appear in a parallel, incompressible flow analysis of this type. For the computed results presented here, shown in Figure 5, $I_{m w 3}$ causes the amplitude to grow faster immediately and not follow the linear growth. To confirm this notion, the calculation used for Figure 6 was repeated for the $M_{j}=1.8$ jet setting $I_{m w 3}=0$. The results are shown in Figure 8. Obviously, without the additional energy input into the coherent modes from non-parallel flow and compressibility effects, the nonlinear amplitude function does initially follow the linear growth before nonlinear effects take over.

The initial amplitude used in the nonlinear calculations also affects the results. This is shown in Figure 9 for the $M_{j}=1.8$ jet where the amplitude is normalized by its initial value. The initial amplitude ranges from $\left|A_{o}\right|^{2}=10^{-6}$ to $10^{-2}$. The normalized linear amplitude line is the same for all initial amplitudes. Hence, only one line is necessary for comparison. The nonlinear amplitude peaks and begins to decay sooner as the initial amplitude increases. The

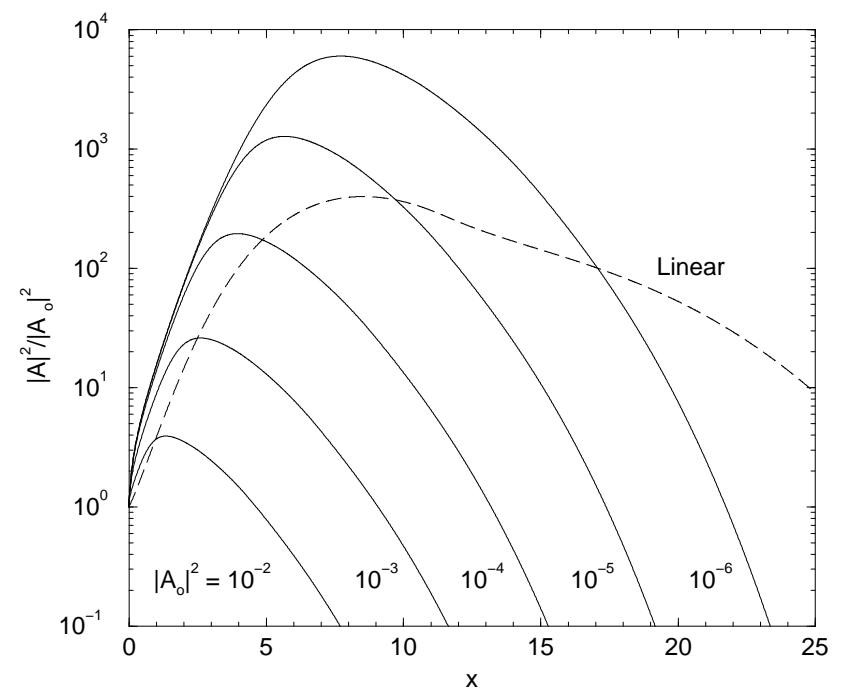

Fig. 9 Amplitude function magnitude comparisons with different initial amplitudes. $\mathrm{M}_{\mathrm{j}}=1.8 ; \mathrm{n}= \pm 1 ; \mathrm{fD} / \overline{\mathrm{U}}_{\mathrm{j}}=0.2$.

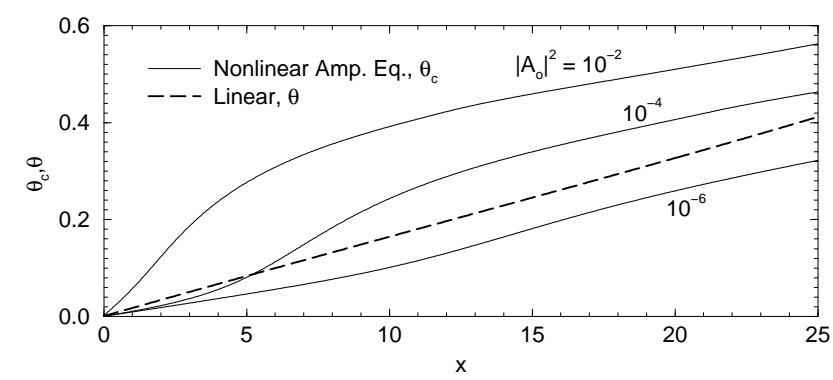

Fig. 10 Shear layer growth comparisons with different initial amplitudes. $\mathrm{M}_{\mathrm{j}}=1.8 ; \mathrm{n}= \pm 1 ; \mathrm{fD} / \overline{\mathrm{U}}_{\mathrm{j}}=0.2$.

stronger initial amplitude leads to the initial increased jet spreading (Figure 10) that results in less total energy being transferred into the mode from the mean flow. The end result is quicker saturation and decay of the mode amplitude. This effect was also noted for the two-dimensional, compressible shear layer. ${ }^{5}$ The weaker initial amplitudes result in delayed spreading, allowing more energy to transfer into the mode even to the point that the amplitude grows larger than the linear amplitude. These results point to an issue that occurs when comparing computed results to measured data. In linear calculations, the unknown initial amplitude is used as a parameter to match data with computations. With nonlinear calculations, every different initial amplitude changes the peak location and shape of the amplitude function. To illustrate, Figure 11 shows qualitative comparisons of both nonlinear and linear amplitude functions to measured data. ${ }^{17}$ The data is from a Mach 1.8 cold jet and the Strouhal number is 0.25 . The measurements include a spatial integration and include the presence of all mode numbers. The calculations are for mode $n= \pm 1$; hence, the comparison is inexact. 

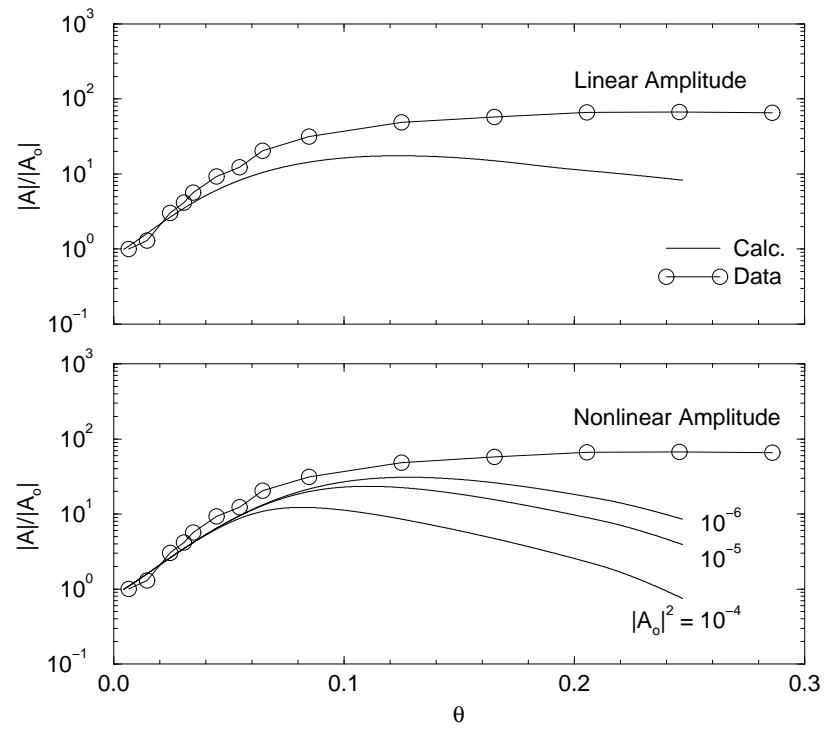

Fig. 11 A comparison of amplitude function magnitude calculations versus measured data. ${ }^{17} \quad \mathrm{M}_{\mathbf{j}}=1.8 ; \mathbf{n}= \pm \mathbf{1}$; $\mathrm{fD} / \overline{\mathrm{U}}_{\mathrm{j}}=0.25$.

\section{CONCLUDING REMARKS}

In this method, the large-scale structure is approximated by a single-frequency, instability wave of a given azimuthal mode number. The fact that the large-structures have a spectrum of frequencies and multiple azimuthal modes has been ignored. Furthermore, the interactions among these modes and with the background, fine-scale turbulence have been neglected. In addition, non-parallel flow effects are not fully taken into account.

Despite these approximations, the results are clearly encouraging indicating qualitative agreements with experiment. The modes initially grow linearly and then saturate as a result of the nonlinear interactions and the flowdivergence effects. Interestingly, the results indicate that the initial growth of the modes becomes larger compared to that of the linear, parallel-flow case due to non-parallel flow and compressibility effects. The results also indicate that the initial level of the disturbance influences the development of the coherent structure and hence the radiated noise field. As such, experimental data on jet noise measurements need to be related to the initial level of the disturbances.

\section{REFERENCES}

1. J. T. C. Liu. Contributions to the understanding of large-scale coherent structures in developing free turbulent shear flows. Adv. App. Mech., 26:183-309, 1988.

2. J. T. C. Liu. Coherent structures in transitional and turbulent free shear flows. Ann. Rev. Fluid Mech., 21:285-315, 1989.
3. R. R. Mankbadi. Dynamics and control of coherent structures in turbulent jets. Appl. Mech. Review, 45:219-248, 1992.

4. R. R. Mankbadi. Transition, Turbulence and Noise: Theory and Applications for Scientists and Engineers. Kluwer, Boston, 1994.

5. K. Lee and J. T. C. Liu. Mixing enhancement in highspeed turbulent shear layers using excited coherent modes. AIAA J., 36:2027-2035, 1998.

6. G. Raman, E. J. Rice, and R. R. Mankbadi. Saturation and the limit of jet mixing enhancement by single frequency plane wave excitation: Experiment and theory. AIAA Paper No. 88-3613, 1988.

7. H. Arbey and J. E. Ffowcs-Williams. Active cancellation of pure tones in an excited jet. J. Fluid Mech., 149:445-454, 1984.

8. T. R. Troutt and D. K. McLaughlin. Experiments on the flow and acoustic properties of a moderateReynolds-number supersonic jet. J. Fluid Mech., 116:123-156, 1982.

9. V. Golubev, R. R. Mankbadi, and M. D. Dahl. Prediction of the acoustic field associated with instability wave source model for a compressible jet. AIAA Paper No. 2002-2455, 2002.

10. S. S. Lee and J. T. C. Liu. Multiple coherent mode interactions in a developing round jet. J. Fluid Mech., 248:383-401, 1993.

11. M. D. Dahl and P. J. Morris. Noise from supersonic coaxial jets, part 1: Mean flow predictions. J. Sound Vib., 200:643-663, 1997.

12. P. J. Morris. The spatial viscous instability of axisymmetric jets. J. Fluid Mech., 77:511-529, 1976.

13. P. J. Morris. Viscous stability of compressible axisymmetric jets. AIAA J., 21:481-482, 1983.

14. C. K. W. Tam and K. C. Chen. A statistical model of turbulence in two-dimensional mixing layers. J. Fluid Mech., 92:303-326, 1979.

15. C. K. W. Tam and D. E. Burton. Sound generated by instability waves of supersonic flows. parts $1 \& 2$. J. Fluid Mech., 138:249-295, 1984.

16. J. R. Scott. Solving ODE initial value problems with implicit Taylor series methods. NASA TM-2000209400, 2000.

17. J. Panda, K. B. M Q. Zaman, and R. G. Seasholtz. Measurement of initial conditions at nozzle exit of high speed jets. AIAA Paper No. 2001-2143, 2001. 
Public reporting burden for this collection of information is estimated to average 1 hour per response, including the time for reviewing instructions, searching existing data sources, gathering and maintaining the data needed, and completing and reviewing the collection of information. Send comments regarding this burden estimate or any other aspect of this collection of information, including suggestions for reducing this burden, to Washington Headquarters Services, Directorate for Information Operations and Reports, 1215 Jefferson Davis Highway, Suite 1204, Arlington, VA 22202-4302, and to the Office of Management and Budget, Paperwork Reduction Project (0704-0188), Washington, DC 20503.

\begin{tabular}{|l|l|r|}
\hline 1. AGENCY USE ONLY (Leave blank) & $\begin{array}{c}\text { 2. REPORT DATE } \\
\text { June } 2002\end{array}$ & $\begin{array}{r}\text { 3. REPORT TYPE AND DATES COVERED } \\
\text { Technical Memorandur }\end{array}$
\end{tabular}

4. TITLE AND SUBTITLE

5. FUNDING NUMBERS

Analysis of Three-Dimensional, Nonlinear Development of Wave-Like Structure in a Compressible Round Jet

6. AUTHOR(S)

WU-708-90-33-00

Milo D. Dahl and Reda R. Mankbadi

7. PERFORMING ORGANIZATION NAME(S) AND ADDRESS(ES)

National Aeronautics and Space Administration

John H. Glenn Research Center at Lewis Field

Cleveland, Ohio 44135-3191

8. PERFORMING ORGANIZATION

REPORT NUMBER

E-13370

9. SPONSORING/MONITORING AGENCY NAME(S) AND ADDRESS(ES)

10. SPONSORING/MONITORING AGENCY REPORT NUMBER

National Aeronautics and Space Administration

Washington, DC 20546-0001

NASA TM-2002-211585

AIAA-2002-2451

\section{SUPPLEMENTARY NOTES}

Prepared for the Eighth Aeroacoustics Conference cosponsored by the American Institute of Aeronautics and Astronautics and the Confederation of European Aerospace Societies, Breckenridge, Colorado, June 17-19, 2002. Milo D. Dahl, NASA Glenn Research Center; and Reda R. Mankbadi, Embry-Riddle Aeronautical University, Daytona Beach, Florida 32114. Responsible person, Milo D. Dahl, organization code 5940, 216-433-3578.

12a. DISTRIBUTION/AVAILABILITY STATEMENT

12b. DISTRIBUTION CODE

Unclassified - Unlimited

Subject Category: 71

Distribution: Nonstandard

Available electronically at http://gltrs.grc.nasa.gov/GLTRS

This publication is available from the NASA Center for AeroSpace Information, 301-621-0390.

13. ABSTRACT (Maximum 200 words)

An analysis of the nonlinear development of the large-scale structures or instability waves in compressible round jets was conducted using the integral energy method. The equations of motion were decomposed into two sets of equations; one set governing the mean flow motion and the other set governing the large-scale structure motion. The equations in each set were then combined to derive kinetic energy equations that were integrated in the radial direction across the jet after the boundary-layer approximations were applied. Following the application of further assumptions regarding the radial shape of the mean flow and the large structures, equations were derived that govern the nonlinear, streamwise development of the large structures. Using numerically generated mean flows, calculations show the energy exchanges and the effects of the initial amplitude on the coherent structure development in the jet.

\section{SUBJECT TERMS}

15. NUMBER OF PAGES

Jet noise; Noise source prediction; Instability waves; Nonlinear instability 17

\begin{tabular}{|c|c|c|}
\hline $\begin{array}{c}\text { 17. SECURITY CLASSIFICATION } \\
\text { OF REPORT } \\
\text { Unclassified }\end{array}$ & $\begin{array}{c}\text { 18. SECURITY CLASSIFICATION } \\
\text { OF THIS PAGE } \\
\text { Unclassified }\end{array}$ & $\begin{array}{c}\text { 19. SECURITY CLASSIFICATION } \\
\text { OF ABSTRACT } \\
\text { Unclassified }\end{array}$ \\
\hline
\end{tabular}

